MANCHESTER

1824

군

Economics

Discussion Paper Series

EDP-1301

Power Sharing and Electoral Equilibrium

\author{
Alejandro Saporiti
}

January 2013

Economics

School of Social Sciences

The University of Manchester

Manchester M13 9PL 


\title{
Power Sharing and Electoral Equilibrium
}

\author{
Alejandro Saporiti*
}

January 14, 2013

\begin{abstract}
We study a model of consensual democracies, where two political parties compete for policy influence in an electoral process, and they share power afterwards proportionally to the number of votes received in the contest. First, we show under mild assumptions that a stable outcome does not always exist in this type of democracies. Second, we offer a condition of symmetry on parties' political motivations which guarantees that a family of proportional power sharing elections satisfy strongly better reply security. This reestablishes the existence of pure strategy equilibrium, though the equilibrium does not need to occur at the median preferred policy. Both findings, particularly the effect of ideology on electoral equilibria and the radicalization of the electoral campaign, stand in sharp contradiction with what happens in winner-take-all democracies, where deterministic voting always leads to the median voter result regardless of parties' motives for running for office.
\end{abstract}

JEL Classification: C72, D71, D72.

Keywords: Electoral system, power sharing, political motivations, Nash equilibrium, strongly better reply security.

\section{Introduction}

The effects of parties' political motivations over electoral equilibria have always received considerable attention in the literature on electoral competition. ${ }^{1}$ Most of the analysis, however, has been done within the spatial model of elections where the winner of a plurality of votes takes full control of policy and government, a framework usually referred to as winner-take-all electoral competition. One of the main conclusion of this literature

\footnotetext{
*University of Manchester; alejandro.saporiti@manchester.ac.uk.

${ }^{1}$ See, for instance, the following recent papers and the references therein: Ball (1999), Saporiti (2008), Callander (2008), Bernhardt, Duggan and Squintani (2009), Drouvelis, Saporiti and Vriend (2011), and Duggan (2012).
} 
is that in the one-dimensional case, parties' ideologies are significant to explain electoral outcomes only if candidates cannot predict voting behavior precisely. Otherwise, under deterministic voting, the median voter result holds regardless of parties' motives for running for office (Duggan 2012).

Winner-take-all are obviously a rather especial type of democracies. In 2012, for instance, Barack Obama won the US presidential election with $51 \%$ of the popular vote against his closest rival Mitt Romney, who got $47.2 \%$. Yet two months after winning office for the second time, President Obama struggled to pass a fiscal package through the American Congress to avoid the so called "fiscal cliff." ${ }^{2}$ After intense negotiations with the opposition, a compromise bill designed to avert the fiscal cliff was finally approved on January 1, 2013. The deal was a "something-for-everyone" mélange. It included low investment tax rates sought by the Republicans and an extension of tax credits for the working poor that were considered among the Democrats' main priorities.

The previous example about the last US presidential election illustrates nicely that in reality, in most democratic countries "the relative power of the majority party for a given election outcome varies with the degree of separation of powers, the organization of chambers, the assignment of committee chairmanships, and institutional rules on agenda setting, allocation of veto powers, and obviously electoral rules," (Herrera et al. 2012). These institutional details shape the mechanism that transforms the votes obtained in the election into actual power to influence policy. Borrowing from Lijphart (1984), we call this second type of polities, where policy making power is shared among parties, consensual democracies. Do political motivations play a distinct role over parties' campaign strategies and the electoral equilibria under this alternative power sharing mechanism?

In this paper, we analyze how parties' interests in power and policy affect the electoral process of consensual democracies. To addresses this matter, we consider a onedimensional, two-party, electoral competition model with mixed motivations, where parties are interested not only in policy but also in their vote shares. We depart from the classical model by assuming that the policy implemented after the contest reflects the platforms of both the majority and the minority party. To be precise, we adopt a "reduced form" mechanism of power sharing where the policy implemented is a combination of the platforms proposed by each party during the electoral campaign weighted by their respective vote shares. ${ }^{3}$ Except for this, the paper remains in all other respects within

\footnotetext{
${ }^{2}$ The term fiscal cliff refers to the sharp decline in the budget deficit that could have occurred in the United States at the beginning of 2013 due to increased taxes and reduced spending as required by previously enacted laws.

${ }^{3}$ The idea is to capture in a stylized way the fact that a party winning with $51 \%$ of the votes will experience more difficulty to carry out its proposal than one winning with say $80 \%$ of the votes. A similar mechanism is used by Ortuño Ortín (1997), Lizzeri and Persico (2001), Persico and Sahuguet (2006), and Herrera, Morelli and Palfrey (2012), to name a few.
} 
the domain of the canonical theory of elections with two parties and deterministic voting.

Besides the simplicity of our framework, the model is shown to be rich enough to deliver new and interesting results. First, an example with symmetric and single-peaked preferences is provided where not only parties' payoff functions are discontinuous and non quasi-concave, but also pure strategy equilibrium fails altogether to exist. We show the root of this instability lies on the fact that consensual democracies (proportionally power sharing electoral games) are not always weakly reciprocally upper semi-continuous (see Definition 5). This electoral instability never takes place under deterministic voting and winner-take-all electoral competition. On the contrary, we already said the latter delivers the median voter result independently of parties' motives for running for office.

Second, to address the problem of equilibrium existence under proportional power sharing, this paper takes advantage of recent developments in the literature on Nash equilibrium in games with discontinuous and non quasi-concave payoffs. The paper shows that in spite of the technical difficulties mentioned before, consensual democracies are always payoff secure (see Definition 4). Moreover, when parties are equally concerned about power, the election game also satisfies a refinement of Reny's (1999) better reply security for compact and non quasi-concave games, called strongly better reply security (see Definition 3). The symmetry of parties' political motivations reestablishes the existence of pure strategy equilibrium. But, as we argue below, the equilibrium does not need to occur at the median preferred policy.

Indeed, the paper analyzes in the symmetric motivation case how the equilibrium platforms vary with the relative interest in policy. The main message is that, contrary to what happens in winner-take-all, ideology matters in consensual democracies even when there is no uncertainty about voters' preferences. In the purely ideological case, each party campaigns in equilibrium on its own ideological side, proposing the most radical platform possible. Then, as their appetite for power increases, they become more moderate, and their electoral policies move towards the center of policy space. The median voter result is eventually recovered when parties split their concerns equally between the two goals, i.e. policy and votes. From that point on, as their concern for power continues raising, the electoral equilibrium of proportionally power sharing election games coincides with the equilibrium of winner-take-all electoral competition.

It is worth noting that, regardless of how much parties care about policy, in the symmetric case the vote shares of each party as well as the implemented policy are the same in both types of institutions. Thus, so long as political parties exhibit a common motivation for competing in elections, consensual and winner-take-all democracies differ only with respect to the campaign strategies of the electoral stage, but the realized outcomes are in both cases the same. In consensual democracies, the dynamic of the 
electoral campaign together with the nature of the power sharing mechanism lead parties to adopt more extreme positions during the electoral process. However, what actually happens afterwards at the post-electoral stage is consistent with the median voter's result. Having said that, recall that when parties do not share a common view about power, that is, in the asymmetric motivation case, the median voter's wish still prevails in winnertake-all democracies, but consensual ones might not even admit a stable outcome in pure strategies.

The rest of the paper is organized as follows. Section 2 presents the model and few auxiliary results, it defines the proportional power sharing election game (consensual democracy), and it discusses an example where pure strategy equilibrium fails to exist. Section 3 focuses on the equilibrium analysis, formally stating and proving the main results of this article. Final remarks and directions for further research appear at the end of the paper in Section 4.

\section{Preliminaries}

Two political parties, indexed by $i=A, B$, compete in an electoral contest by simultaneously and independently announcing a policy platform $x_{i} \in X=[0,1]$. The electorate is made up of a continuum of voters of mass equal to 1 . Voters differ in a single parameter $\theta$, which is distributed over $[0,1]$ according with a probability distribution function $F$. A voter of type $\theta$ has preferences over $X$ represented by a real-valued utility function $U(x, \theta)$. We assume that both $F$ and $U(x, \theta)$ are common knowledge.

For every pair of announced policies $\left(x_{A}, x_{B}\right) \in X \times X$, each voter votes deterministically for the party whose proposal offers the highest utility, voting for the two alternatives with equal probabilities when indifferent. ${ }^{4}$ The policy implemented after the election, denoted by $x\left(x_{A}, x_{B}\right)$, is given by

$$
x\left(x_{A}, x_{B}\right)=\rho_{A}\left(x_{A}, x_{B}\right) \cdot x_{A}+\rho_{B}\left(x_{A}, x_{B}\right) \cdot x_{B},
$$

where $\rho_{i}\left(x_{A}, x_{B}\right) \in[0,1]$ denotes party $i$ 's vote share, and $\sum_{i} \rho_{i}\left(x_{A}, x_{B}\right)=1$. As was mentioned in the Introduction, a expression like (1) is employed in some important papers of political economy, including Lizzeri and Persico (2001) and Persico and Sahuguet (2006). Here we study the implication of that assumption for the existence of electoral equilibrium and the effects of ideology.

We suppose throughout the analysis that the following assumptions holds.

\footnotetext{
${ }^{4}$ Notice that by Assumption 4 below, for all $x_{A} \neq x_{B}, F\left(\left\{\theta \in[0,1]: U\left(x_{A}, \theta\right)=U\left(x_{B}, \theta\right)\right\}\right)=0$.
} 
Ass $1 F(\theta)$ is twice continuously differentiable, with continuous density $F^{\prime}(\cdot) \equiv f(\cdot)$ and $F\left(\theta_{M}\right)=1 / 2$ for some $\theta_{M} \in(0,1)$.

Ass $2 U(x, \theta)$ is continuous in both arguments, strictly concave in $x$, and differentiable in $x$ everywhere, except possibly at $\hat{x}(\theta)=\arg \max _{x \in X} U(x, \theta)$, with $\lim _{x \rightarrow 0} U(x, \theta) \in \mathbb{R}$ and $\lim _{x \rightarrow 1} U(x, \theta) \in \mathbb{R}$.

Ass $3 \hat{x}(\theta)$ is strictly increasing in $\theta$, with $\hat{x}(0)=0$ and $\hat{x}(1)=1$.

Ass 4 For all $x, y \in X$, with $x \neq y$, there exists at most one type $\bar{\theta} \in[0,1]$ such that $U(x, \bar{\theta})-U(y, \bar{\theta})=0$.

Let $\bar{\theta}\left(x_{A}, x_{B}\right)$ be implicitly defined by $U\left(x_{A}, \bar{\theta}\left(x_{A}, x_{B}\right)\right)=U\left(x_{B}, \bar{\theta}\left(x_{A}, x_{B}\right)\right)$.

Ass $5 \bar{\theta}\left(x_{A}, x_{B}\right)$ is differentiable with respect to $x_{A}$ and $x_{B}\left(x_{A} \neq x_{B}\right)$.

Ass $6 \frac{F\left(\bar{\theta}\left(x_{i}, x_{-i}\right)\right)}{\frac{\partial F\left(\bar{\theta}\left(x_{i}, x_{-i}\right)\right)}{\partial x_{i}}}$ is nondecreasing in $x_{i}$, and $\frac{1-F\left(\bar{\theta}\left(x_{i}, x_{-i}\right)\right)}{\frac{\partial F\left(\bar{\theta}\left(x_{i}, x_{-}\right)\right)}{\partial x_{-i}}}$ is nonincreasing in $x_{-i}{ }^{5}$

It is easy to show that under Ass 6 both $\ln \left(F\left(\bar{\theta}\left(x_{i}, x_{-i}\right)\right)\right)$ and $\ln \left(1-F\left(\bar{\theta}\left(x_{i}, x_{-i}\right)\right)\right)$ are concave in $x_{i}{ }^{6}$ Therefore, $F\left(\bar{\theta}\left(x_{i}, x_{-i}\right)\right)$ and $1-F\left(\bar{\theta}\left(x_{i}, x_{-i}\right)\right)$ are quasi-concave in $x_{i}$. Moreover, for any pair of distinct policies, the structure imposed so far allows to split the electorate into two disjoint subsets, namely, the set of voters that supports party $A$ and the set that votes for party $B$. This result is stated formally in Lemma 1.

Lemma 1 For any pair of policies $\left(x_{A}, x_{B}\right) \in X \times X$, with $x_{A} \neq x_{B}, \rho_{i}\left(x_{A}, x_{B}\right)=$ $F\left(\left[0, \bar{\theta}\left(x_{A}, x_{B}\right)\right]\right)$ and $\rho_{-i}\left(x_{A}, x_{B}\right)=F\left(\left[\bar{\theta}\left(x_{A}, x_{B}\right), 1\right]\right)$ for some $i=A, B$.

Proof Without loss of generality, assume $x_{A}<x_{B}$. By Ass 2 and Ass 3, $U\left(x_{A}, 0\right)-$ $U\left(x_{B}, 0\right)>0$ and $U\left(x_{A}, 1\right)-U\left(x_{B}, 1\right)<0$. Thus, since by Ass $2 U\left(x_{A}, \theta\right)-U\left(x_{B}, \theta\right)$ is continuous in $\theta$, by the intermediate value theorem there must exist $\tilde{\theta} \in(0,1)$ such that $U\left(x_{A}, \tilde{\theta}\right)-U\left(x_{B}, \tilde{\theta}\right)=0$. By Ass $4, \tilde{\theta}$ is unique; thereby by definition, $\tilde{\theta}=\bar{\theta}\left(x_{A}, x_{B}\right)$.

Consider next any type $\theta^{\prime} \in(0, \tilde{\theta})$, and suppose by way of contradiction that $U\left(x_{A}, \theta^{\prime}\right)-U\left(x_{B}, \theta^{\prime}\right)<0$. Repeating the argument employed before, there must exist a type $\widetilde{\widetilde{\theta}} \in\left(0, \theta^{\prime}\right)$ such that $U\left(x_{A}, \widetilde{\widetilde{\theta}}\right)-U\left(x_{B}, \widetilde{\widetilde{\theta}}\right)=0$, which provides the desired contradiction with Ass 4 (because $\widetilde{\tilde{\theta}}<\tilde{\theta})$. Hence, for every $\theta \in(0, \tilde{\theta}), U\left(x_{A}, \theta\right)-U\left(x_{B}, \theta\right)>0$. Following an analogous reasoning, it can be shown that for every $\theta \in(\tilde{\theta}, 1), U\left(x_{A}, \theta\right)-U\left(x_{B}, \theta\right)<0$. Thus, $\rho_{A}\left(x_{A}, x_{B}\right)=F\left(\left[0, \bar{\theta}\left(x_{A}, x_{B}\right)\right]\right)$ and $\rho_{B}\left(x_{A}, x_{B}\right)=F\left(\left[\bar{\theta}\left(x_{A}, x_{B}\right), 1\right]\right)$.

\footnotetext{
${ }^{5}$ Hereafter, it is understood that the index $-i$ denotes $B$ if $i=A$ and $A$ if $i=B$.

${ }^{6}$ See Bagnoli and Bergstrom (2005) for applications of log-concave probability functions into economics and political science.
} 
Using Lemma 1, we can determine for any pair of distinct policies, the response of each party's vote share to changes in its policy platform. In words, we show in Lemma 2 that as one party moves its platform toward that of (resp., away from) its opponent, it increases (resp., decreases) its vote share.

Lemma 2 For any $x_{i}<x_{-i}$, (a) $\frac{\partial \rho_{i}\left(x_{i}, x_{-i}\right)}{\partial x_{i}}>0$, and (b) $\frac{\partial \rho_{-i}\left(x_{i}, x_{-i}\right)}{\partial x_{-i}}<0$.

Proof Consider any two policies $x_{i}, x_{-i} \in X$ such that $x_{i}<x_{-i}$. Let $x_{i}^{\prime}=x_{i}+\delta$, with $\delta>0$ and $x_{i}^{\prime}<x_{-i}$. Assume by contradiction that $\bar{\theta}\left(x_{i}, x_{-i}\right)>\bar{\theta}\left(x_{i}^{\prime}, x_{-i}\right)$. Since $\theta$ is continuously distributed on $X$, there exists $\tilde{\theta}$ between $\bar{\theta}\left(x_{i}, x_{-i}\right)$ and $\bar{\theta}\left(x_{i}^{\prime}, x_{-i}\right)$ such that

$$
U\left(x_{-i}, \tilde{\theta}\right)>U\left(x_{i}^{\prime}, \tilde{\theta}\right)
$$

where the last inequality follows from the fact that, by Lemma $1, \rho_{i}\left(x_{i}^{\prime}, x_{-i}\right)=$ $F\left(\left[0, \bar{\theta}\left(x_{i}^{\prime}, x_{-i}\right)\right]\right)$. By Ass $2, \hat{x}(\tilde{\theta})>x_{i}^{\prime}$. Otherwise, if $\hat{x}(\tilde{\theta}) \leq x_{i}^{\prime}$, strict concavity (singlepeakedness) of $U(\cdot, \tilde{\theta})$ together with $x_{i}^{\prime}<x_{-i}$ would imply that $U\left(x_{-i}, \tilde{\theta}\right)<U\left(x_{i}^{\prime}, \tilde{\theta}\right)$, contradicting (2). Moreover, $\hat{x}(\tilde{\theta})>x_{i}^{\prime}>x_{i}$ implies that $U\left(x_{i}^{\prime}, \tilde{\theta}\right)>U\left(x_{i}, \tilde{\theta}\right)$; and combining this with $(2)$, we get that $U\left(x_{-i}, \tilde{\theta}\right)>U\left(x_{i}, \tilde{\theta}\right)$, contradicting that by Lemma 1 $\rho_{i}\left(x_{i}, x_{-i}\right)=F\left(\left[0, \bar{\theta}\left(x_{i}, x_{-i}\right)\right]\right)$.

Next, suppose $\bar{\theta}\left(x_{i}, x_{-i}\right)=\bar{\theta}\left(x_{i}^{\prime}, x_{-i}\right)$. By definition of $\bar{\theta}\left(x_{i}^{\prime}, x_{-i}\right)$,

$$
U\left(x_{i}^{\prime}, \bar{\theta}\left(x_{i}^{\prime}, x_{-i}\right)\right)=U\left(x_{-i}, \bar{\theta}\left(x_{i}^{\prime}, x_{-i}\right)\right)
$$

thus, $x_{i}^{\prime}<\hat{x}\left(\bar{\theta}\left(x_{i}^{\prime}, x_{-i}\right)\right)<x_{-i}$. By Ass $3, \hat{x}\left(\bar{\theta}\left(x_{i}, x_{-i}\right)\right)=\hat{x}\left(\bar{\theta}\left(x_{i}^{\prime}, x_{-i}\right)\right)$; and by definition,

$$
U\left(x_{i}, \bar{\theta}\left(x_{i}, x_{-i}\right)\right)=U\left(x_{-i}, \bar{\theta}\left(x_{i}, x_{-i}\right)\right) .
$$

Notice that the right-hand side of (3) and (4) are equal because by hypothesis the types are identical. Thus, we have that $U\left(x_{i}^{\prime}, \bar{\theta}\left(x_{i}, x_{-i}\right)\right)=U\left(x_{i}, \bar{\theta}\left(x_{i}, x_{-i}\right)\right)$, which stands in contradiction with Ass 2 (recall $x_{i}<x_{i}^{\prime}<\hat{x}\left(\bar{\theta}\left(x_{i}, x_{-i}\right)\right)$ ). Hence, for any $x_{i}<x_{i}^{\prime}<$ $x_{-i} \in X, \bar{\theta}\left(x_{i}, x_{-i}\right)<\bar{\theta}\left(x_{i}^{\prime}, x_{-i}\right)$; and since $F(\cdot)$ is an increasing function $F\left(\bar{\theta}\left(x_{i}, x_{-i}\right)\right)<$ $F\left(\bar{\theta}\left(x_{i}^{\prime}, x_{-i}\right)\right)$. Moving terms to the right hand side, dividing by $\delta>0$, and taking the limit as $\delta$ goes to zero (which exists by Ass 4 ), we have that

$$
\lim _{\delta \rightarrow 0} \frac{F\left(\bar{\theta}\left(x_{i}+\delta, x_{-i}\right)\right)-F\left(\bar{\theta}\left(x_{i}, x_{-i}\right)\right)}{\delta}>0
$$

which delivers the result stated in (a). The proof of (b) follows a similar argument.

Parties care about votes and policy. Formally, for every $\left(x_{A}, x_{B}\right) \in X \times X$ the payoff 
functions for parties $A$ and $B$ are, respectively,

$$
\Pi_{A}\left(x_{A}, x_{B}\right)=\lambda_{A} \cdot \rho_{A}\left(x_{A}, x_{B}\right)+\left(1-\lambda_{A}\right) \cdot U_{A}\left[x\left(x_{A}, x_{B}\right)\right]
$$

and

$$
\Pi_{B}\left(x_{A}, x_{B}\right)=\lambda_{B} \cdot \rho_{B}\left(x_{A}, x_{B}\right)+\left(1-\lambda_{B}\right) \cdot U_{B}\left[x\left(x_{A}, x_{B}\right)\right],
$$

where $\lambda_{i} \in(0,1)$ stands for party $i$ 's weight on his vote share, and $U_{i}(\cdot) \equiv U\left(\cdot, \theta_{i}\right)$ for some $\theta_{i} \in[0,1]$. We assume that party $A$ (resp., $B$ ) represents a voter type located on the left-hand (resp., right-hand) side of the median type, i.e., $\theta_{A}<\theta_{M}<\theta_{B}$; and we identify the half-open interval $\left[0, \theta_{M}\right)$ (resp., $\left.\left(\theta_{M}, 1\right]\right)$ with the left-wing (resp., right-wing) ideological side. Notice that for any $x_{i}<x_{-i}\left(\right.$ resp. $\left.x_{i}>x_{-i}\right)$, the function $U_{i}\left(x\left(x_{i}, x_{-i}\right)\right)$ is single-peaked in $x_{i}$ (Ortuño Ortín (1997, p.433). Therefore, by Lemma $2, \Pi_{i}\left(x_{i}, x_{-i}\right)$ is single-peaked on $x_{i}<x_{-i}$ (resp. $x_{i}>x_{-i}$ ) as well. This information about the shape of the payoff functions will be useful in the proof of Proposition 2. Similarly, the result that follows will be employed to prove Proposition 1.

Lemma 3 Fix $i=A, B$ and consider any profile $\bar{x}=\left(\bar{x}_{i}, \bar{x}_{-i}\right) \in X \times X$. If either (i) $\bar{x}_{i} \neq \bar{x}_{-i}$, (ii) $\bar{x}_{i}=\hat{x}\left(\theta_{M}\right)$, or (iii) $\lambda_{i}=0$, then $\Pi_{i}\left(\bar{x}_{i}, \cdot\right)$ is continuous in $x_{-i}$ at $\bar{x}_{-i}$.

Proof Consider first the case where $\bar{x}_{i} \neq \bar{x}_{-i}$. Without loss of generality, suppose $\bar{x}_{i}<$ $\bar{x}_{-i}$. By Lemma $1, \rho_{i}\left(\bar{x}_{i}, \bar{x}_{-i}\right)=F\left(\left[0, \bar{\theta}\left(\bar{x}_{i}, \bar{x}_{-i}\right)\right]\right)$, where $\bar{\theta}\left(\bar{x}_{i}, \bar{x}_{-i}\right)$ is implicitly defined by the equation $U\left(\bar{x}_{i}, \bar{\theta}\left(\bar{x}_{i}, \bar{x}_{-i}\right)\right)-U\left(\bar{x}_{-i}, \bar{\theta}\left(\bar{x}_{i}, \bar{x}_{-i}\right)\right)=0$, which has a unique solution in $\theta$ by Ass 2-4. Since $F(\cdot)$ is a continuous distribution function, by Ass 5 (i.e., continuity of $\left.\bar{\theta}\left(\bar{x}_{i}, \cdot\right)\right)$ the functions $\rho_{i}\left(\bar{x}_{i}, \cdot\right), x\left(\bar{x}_{i}, \cdot\right)$ and $U_{i}\left(x\left(\bar{x}_{i}, \cdot\right)\right)$ are all continuous in $x_{-i}$ at $\bar{x}_{-i}$. Hence, $\Pi_{i}\left(\bar{x}_{i}, \cdot\right)$ is continuous in $x_{-i}$ at $\bar{x}_{-i}$ as well.

Next suppose $\bar{x}_{i}=\hat{x}\left(\theta_{M}\right)$. If $\bar{x}_{-i} \neq \hat{x}\left(\theta_{M}\right)$, then we can use the previous argument to derive the desired result. Thus, assume $\bar{x}_{-i}=\hat{x}\left(\theta_{M}\right)$. Note that $\rho_{i}\left(\bar{x}_{i}, \bar{x}_{-i}\right)=$ $1 / 2=F\left(\left[0, \theta_{M}\right]\right)$. Consider a sequence $\left\{x_{-i}^{n}\right\} \rightarrow \hat{x}\left(\theta_{M}\right)$. For any $x_{-i}^{n} \neq \hat{x}\left(\theta_{M}\right)$, let $\bar{\theta}^{n} \equiv \bar{\theta}\left(\hat{x}\left(\theta_{M}\right), x_{-i}^{n}\right)$. Notice that by Ass 2-4, $\bar{\theta}^{n}$ is well defined. Suppose, without loss of generality, that $x_{-i}^{n}<\hat{x}\left(\theta_{M}\right)$. Clearly, $\hat{x}\left(\bar{\theta}^{n}\right) \neq x_{-i}^{n}$ (resp., $\neq \hat{x}\left(\theta_{M}\right)$ ). Moreover, if $\hat{x}\left(\bar{\theta}^{n}\right)<x_{-i}^{n}$ (resp., $\left.\hat{x}\left(\bar{\theta}^{n}\right)>\hat{x}\left(\theta_{M}\right)\right)$, then Ass 2 would imply that $U\left(x_{-i}^{n}, \bar{\theta}^{n}\right)>$ $U\left(\hat{x}\left(\theta_{M}\right), \bar{\theta}^{n}\right)$ (resp., $\left.U\left(x_{-i}^{n}, \bar{\theta}^{n}\right)<U\left(\hat{x}\left(\theta_{M}\right), \bar{\theta}^{n}\right)\right)$, contradicting the definition of $\bar{\theta}^{n}$. Thus, $x_{-i}^{n}<\hat{x}\left(\bar{\theta}^{n}\right)<\hat{x}\left(\theta_{M}\right)$; and consequently $\left\{\hat{x}\left(\bar{\theta}^{n}\right)\right\} \rightarrow \hat{x}\left(\theta_{M}\right)$ as $x_{-i}^{n} \rightarrow \hat{x}\left(\theta_{M}\right)$. Moreover, $\left\{\bar{\theta}^{n}\right\} \rightarrow \theta_{M}$ as well, since otherwise we would have that $\lim _{n \rightarrow \infty} \hat{x}\left(\bar{\theta}^{n}\right) \equiv \hat{x}\left(\bar{\theta}^{\infty}\right)=\hat{x}\left(\theta_{M}\right)$ but $\lim _{n \rightarrow \infty} \bar{\theta}^{n} \equiv \bar{\theta}^{\infty} \neq \theta_{M}$, which stands in contradiction with Ass 3. ${ }^{7}$ Therefore, $\bar{\theta}\left(\hat{x}\left(\theta_{M}\right), x_{-i}^{n}\right) \rightarrow \theta_{M}$ as $x_{-i}^{n} \rightarrow \hat{x}\left(\theta_{M}\right)$, and $F\left(\bar{\theta}\left(\hat{x}\left(\theta_{M}\right), x_{-i}^{n}\right)\right) \rightarrow 1 / 2$. Hence, $\rho_{i}\left(\hat{x}\left(\theta_{M}\right), \cdot\right)$ is continuous in $x_{-i}$ at $\hat{x}\left(\theta_{M}\right)$; and so are $x\left(\hat{x}\left(\theta_{M}\right), \cdot\right), U_{i}\left(x\left(\hat{x}\left(\theta_{M}\right), \cdot\right)\right)$, and $\Pi_{i}\left(\hat{x}\left(\theta_{M}\right), \cdot\right)$.

\footnotetext{
${ }^{7}$ Notice that $\lim _{n \rightarrow \infty} \bar{\theta}^{n} \equiv \lim _{x_{-i}^{n} \rightarrow \hat{x}\left(\bar{\theta}_{M}\right)} \bar{\theta}\left(\hat{x}\left(\theta_{M}\right), x_{-i}^{n}\right)$ exists because $\bar{\theta}\left(\hat{x}\left(\theta_{M}\right), \cdot\right)$ is by Ass 5 continuous on $x_{-i} \neq \hat{x}\left(\theta_{M}\right)$, and so $\left\{\bar{\theta}\left(\hat{x}\left(\theta_{M}\right), x_{-i}^{n}\right)\right\}$ converges as $n \rightarrow \infty$.
} 
Finally, suppose $\lambda_{i}=0$, so that $\Pi_{i}\left(\bar{x}_{i}, \bar{x}_{-i}\right)=U_{i}\left(x\left(\bar{x}_{i}, \bar{x}_{-i}\right)\right)$. Recall that $x\left(\bar{x}_{i}, \bar{x}_{-i}\right)=$ $\rho_{i}\left(\bar{x}_{i}, \bar{x}_{-i}\right) \cdot\left[\bar{x}_{i}-\bar{x}_{-i}\right]+\bar{x}_{-i}$. As was shown before, if $\bar{x}_{i} \neq \bar{x}_{-i}$, then $x\left(\bar{x}_{i}, \cdot\right)$ is continuous in $x_{-i}$ at $\bar{x}_{-i}$ and we are done. Otherwise, consider a sequence $x^{n}=\left(x_{i}^{n}, x_{-i}^{n}\right) \subseteq X \times X$, such that $\left(x_{i}^{n}, x_{-i}^{n}\right) \rightarrow\left(\bar{x}_{i}, \bar{x}_{-i}\right)$. Taking the limit of $x\left(x_{i}^{n}, x_{-i}^{n}\right)$ as $\left(x_{i}^{n}, x_{-i}^{n}\right) \rightarrow\left(\bar{x}_{i}, \bar{x}_{-i}\right)$, we have that $x\left(x_{i}^{n}, x_{-i}^{n}\right) \rightarrow \bar{x}_{-i}=x\left(\bar{x}_{i}, \bar{x}_{-i}\right)$, since $\rho_{i}\left(x_{i}^{n}, x_{-i}^{n}\right)$ is bounded and $\left[x_{i}^{n}-x_{-i}^{n}\right]$ converges to zero as $x^{n} \rightarrow \bar{x}$. Therefore, $x\left(\bar{x}_{i}, \cdot\right)$ is continuous in $x_{-i}$ at $\bar{x}_{-i}$, and so is the payoff function $\Pi_{i}\left(\bar{x}_{i}, \cdot\right)$.

Let $\mathcal{G}=\left(X, \Pi_{i}\right)_{i=A, B}$ be a proportionally power sharing election game that satisfies Ass (1)-(6). A pure strategy equilibrium (PSE) for $\mathcal{G}$ is a pair of policy platforms $\left(x_{A}^{*}, x_{B}^{*}\right) \in X \times X$ such that $\Pi_{A}\left(x_{A}^{*}, x_{B}^{*}\right) \geq \Pi_{A}\left(y, x_{B}^{*}\right)$ and $\Pi_{B}\left(x_{A}^{*}, x_{B}^{*}\right) \geq \Pi_{B}\left(x_{A}^{*}, y\right)$ for all $y \in X$. When both parties care only about their vote shares, i.e., when $\lambda_{i}=1$ for all $i=A, B$, such equilibrium is known to exist and to coincide with the median voter's ideal policy $\hat{x}\left(\theta_{M}\right)$ (see, for instance, Roemer 2001, p.26). Likewise, in the other extreme case, that is, in the purely ideological case where $\lambda_{i}=0$ for all $i=A, B$, Ortuño Ortín (1997) shows that $\Pi_{i}$ is continuous in $x_{i}$; and he applies Brower's Fixed Point Theorem to conclude that a pure strategy equilibrium always exists as well.

In contrast with these positive results associated with the extreme cases of vote and policy motivation, Example 1 below illustrates that when $\lambda_{i} \in(0,1)$ not only the conditional payoff functions $\Pi_{i}\left(\cdot, x_{-i}\right)$ are discontinuous and might not be quasi-concave, but also that $\mathcal{G}$ does not necessarily possess an equilibrium in pure strategies. This stands in sharp contrast with what happens in winner-take-all elections under similar circumstances, i.e., with mixed motivations and without electoral uncertainty. That model admits in the one-dimensional case a unique equilibrium in pure strategies in which both platforms coincide with the median voter's preferred policy (Duggan 2012, p.16). Thus, our example suggests that mixed motivations alone can't be blamed here for the lack of stability. The power sharing mechanism plays its role too!

Example 1 (Uniform distribution \& Euclidean preferences) Suppose $\theta$ is uniformly distributed on $[0,1]$ and let $U(x, \theta)=-\|x-\theta\|$. Then, $\hat{x}(\theta)=\theta$ and $\bar{\theta}\left(x_{A}, x_{B}\right)=\frac{x_{A}+x_{B}}{2}=F\left(\bar{\theta}\left(x_{A}, x_{B}\right)\right)$, meaning that Ass (1)-(6) hold. Assume that $\lambda_{A}=\frac{11}{21}$ and $\lambda_{B}=\frac{4}{10}$, with $\theta_{A}=\frac{2}{10}$ and $\theta_{B}=\frac{8}{10}$, and consider a PSE candidate $x_{A}^{*}<x_{B}^{*}$. As Figs. 1 and 2 illustrate, near the critical points the conditional payoff functions are continuous and differentiable; thereby the first order conditions imply that $x_{A}^{*}=\frac{11}{20}$ and $x_{B}^{*}=\frac{2}{3}$.

Note in Fig. 2 that although party $B$ 's second order condition is satisfied at the stationary point $x_{B}^{*}=2 / 3$, his conditional payoff function $\Pi_{B}\left(x_{A}^{*}, \cdot\right)$ is neither continuous nor quasi-concave on $[0,1]$. Moreover, the local maximizer $x_{B}^{*}=2 / 3$ is not $B$ 's best response to $x_{A}^{*}=11 / 20$. Indeed, party $B$ can do better by undercutting $11 / 20$ from 
below, which leads to an empty best response function for that value of $x_{A}$. Hence, there is no equilibrium in pure strategies with $x_{A}^{*}<x_{B}^{*}$.

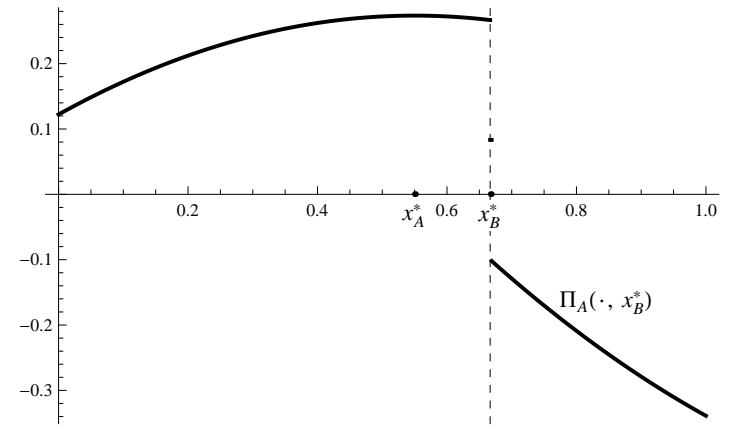

Figure 1: Party $A$ 's conditional payoff function given $x_{B}^{*}=\frac{2}{3}$.

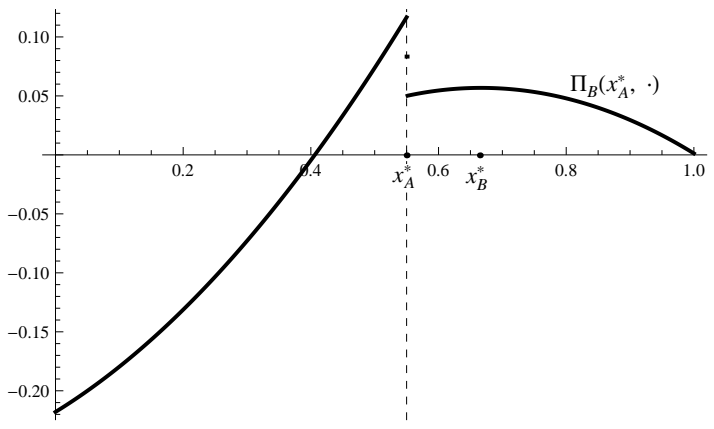

Figure 2: Party $B$ 's conditional payoff function given $x_{A}^{*}=\frac{11}{20}$.

Regarding the existence of PSE with $x_{A}^{*} \geq x_{B}^{*}$, first the profile of median ideal policies $(1 / 2,1 / 2)$ can't be a PSE unless $\lambda_{i} \geq 1 / 2$ for all $i=A, B$. In this example, this condition fails to hold for party $B$, which has a profitable deviation to $1 / 2+\delta$ for $\delta>0$ sufficiently small. Similarly, there is no PSE with $x_{A}^{*}=x_{B}^{*}=x \neq 1 / 2$. For instance, if $x>1 / 2$, then party $A$ has a profitable deviation to the left of $x$. Finally, there is no equilibrium with $x_{A}^{*}>x_{B}^{*}$ either, because: (i) If $0<x_{B}^{*} \leq \theta_{A}$, then $B$ can profitable deviate towards $x_{A}^{*}$; (ii) If $\theta_{A}<x_{B}^{*}<\theta_{M}$, party $A$ can continuously increase his payoff by moving closer to $x_{B}^{*}$; and (iii) If $\theta_{M} \leq x_{B}^{*}<1$, then $A$ can increase his payoff by tying with $B$ at $x_{B}^{*}$. This together with the comments in the previous paragraph shows that the election game under consideration has no equilibrium in pure strategies.

The example discussed above shows how sensible is Ortuño Ortín's (1997) analysis of proportional representation elections to the hypothesis that parties care only about policy $\left(\lambda_{i}=0\right)$. This is not just because, as we saw, pure strategy equilibrium might fail to exist under mixed motivations $\left(\lambda_{i} \in(0,1)\right)$. More importantly, in the latter case the conditional payoff functions are not continuous and may violate quasi-concavity, and this invalidates Ortuño Ortín's (1997) approach to equilibrium existence, which is based on the direct application of a fixed point argument. In the next section, we address this problem using the recent developments in the literature on the existence of pure Nash equilibria in discontinuous and non quasi-concave strategic games.

\section{Equilibrium Analysis}

The aim of this section is to provide a sufficient condition that generalizes Ortuño Ortín's (1997) existence result to a broader class of proportionally power sharing election games, 
in which parties are concerned not only with policy, but also with their vote share. Roughly speaking, the strategy consists in establishing a restriction on the parameters $\lambda_{i}$ such that the game satisfies a property called strongly better reply security (SBRS). This property, recently proposed by Bich (2009), generalizes Reny's (1999) better reply security in a way that will become clear shortly. Technically, it guarantees that every compact game admits a Nash equilibrium in pure strategies, regardless of whether the game is quasi-concave or not (Theorem 3.2, Bich 2009, p.401). ${ }^{8}$ As is shown in Example 3 , in general the proportionally power sharing election game is not SBRS; hence the need to restrict $\lambda_{i}$.

To proceed, we introduce first a local index of non quasi-concavity for the conditional payoff functions of $\mathcal{G}$. Fix $x_{-i} \in X$ and recall that the function $\Pi_{i}\left(\cdot, x_{-i}\right)$ is quasi-concave on a nonempty and convex subset $Y \subseteq X$ if for all $y, y^{\prime} \in Y$ and all $\alpha \in[0,1]$, one has that $\Pi_{i}\left(\alpha y+(1-\alpha) y^{\prime}, x_{-i}\right) \geq \min \left\{\Pi_{i}\left(y, x_{-i}\right), \Pi_{i}\left(y^{\prime}, x_{-i}\right)\right\}$. Equivalently, following Bich (2009), one could say that $\Pi_{i}\left(\cdot, x_{-i}\right)$ is quasi-concave on $Y$ if for every natural number $n \in \mathbb{N}$ and every pair $(\alpha, y) \in \Delta^{n-1} \times Y^{n}$,

$$
\Pi_{i}\left(\alpha \cdot y, x_{-i}\right) \geq \min \left\{\Pi_{i}\left(y^{1}, x_{-i}\right), \ldots, \Pi_{i}\left(y^{n}, x_{-i}\right)\right\}
$$

where $\Delta^{n-1}=\left\{\left(\alpha^{1}, \ldots, \alpha^{n}\right) \in \mathbb{R}_{+}^{n}: \sum_{k=1}^{n} \alpha^{k}=1\right\}$ is the simplex of $\mathbb{R}^{n}$. Next, we measure how much $(7)$ can be false at $\hat{y} \in Y$. This provides a measure of the lack of quasi-concavity around the selected point. To do that, define the mapping $\Phi_{i}\left(\cdot, x_{-i}\right): Y^{n} \rightarrow \mathbb{R}$ as

$$
\forall \hat{y} \in Y, \Phi_{i}\left(\hat{y}, x_{-i}\right)=\sup \left\{\min \left\{\Pi_{i}\left(y^{1}, x_{-i}\right), \ldots, \Pi_{i}\left(y^{n}, x_{-i}\right)\right\}-\Pi_{i}\left(\hat{y}, x_{-i}\right)\right\}
$$

where the supremum is taken over all $n \in \mathbb{N}$ and all families $\left\{y^{1}, \ldots, y^{n}\right\} \subseteq Y$ such that $\hat{y} \in \operatorname{co}\left(\left\{y^{1}, \ldots, y^{n}\right\}\right) .^{9}$ (Bear in mind that $x_{-i}$ has been fixed above.)

Definition 1 The mapping $\sigma_{i}$, defined as $\sigma_{i}\left(y, x_{-i}\right)=\limsup _{\hat{y} \rightarrow y} \Phi_{i}\left(\hat{y}, x_{-i}\right)$ for all $y \in Y \subseteq$ $X$, measures the lack of quasi-concavity of $\Pi_{i}\left(\cdot, x_{-i}\right)$ at $y$.

As is noted in Bich (2009), for every $y \in Y, \sigma_{i}\left(y, x_{-i}\right) \geq 0$. Moreover, a compact game is quasi-concave if and only if, for all $x_{-i}, \sigma_{i}\left(\cdot, x_{-i}\right)$ is equal to zero for every player $i$ at every possible point on the domain of $\Pi_{i}\left(\cdot, x_{-i}\right)$. Finally, by definition, $\sigma_{i}$ is upper semi-continuous.

Let $\tilde{\Pi}_{i}\left(x_{i}, x_{-i}\right)=\inf \left\{\pi_{i}\left(x_{i}, x_{-i}\right): \pi_{i}\left(\cdot, x_{-i}\right): X \rightarrow \mathbb{R}\right.$ quasi-concave, $\Pi_{i}\left(\cdot, x_{-i}\right) \leq$ $\left.\pi_{i}\left(\cdot, x_{-i}\right)\right\}$ be the quasi-concave hull of $\Pi_{i}\left(x_{i}, x_{-i}\right)$ (with respect to party $i$ 's strategy).

\footnotetext{
${ }^{8} \mathrm{~A}$ strategic game is said to be compact if the pure strategy set of each player is a nonempty, compact and convex subset of a topological vector space, and his payoff function is bounded. The game is said to be quasi-concave if each player's conditional payoff function is quasi-concave in his own strategy.

${ }^{9}$ In the sequel, for every set $S, \operatorname{co}(S)$ stands for the convex hull of $S$.
} 
Then, for any $y \in Y, \Phi_{i}\left(y, x_{-i}\right)=\tilde{\Pi}_{i}\left(y, x_{-i}\right)-\Pi_{i}\left(y, x_{-i}\right)$ (Prop 2.2, Bich 2009, p.398). Thus, $\sigma_{i}$ can be thought as the upper semi-continuous regularization of the distance between $\Pi_{i}\left(\cdot, x_{-i}\right)$ and its quasi-concave hull (Remark 2.2, Bich 2009, p.399).

Example 2 (cont. Example 1) The quasi-concave hull and the index of lack of quasiconcavity for the conditional payoff function represented in Fig. 2 are illustrated in Figs. 3 and 4, respectively. Fig. 3 overlaps the quasi-concave hull (in red) over the original payoff function (in black). These functions differ only over the open interval $(11 / 20,2 / 3)$, by the amount represented in Fig. 4.

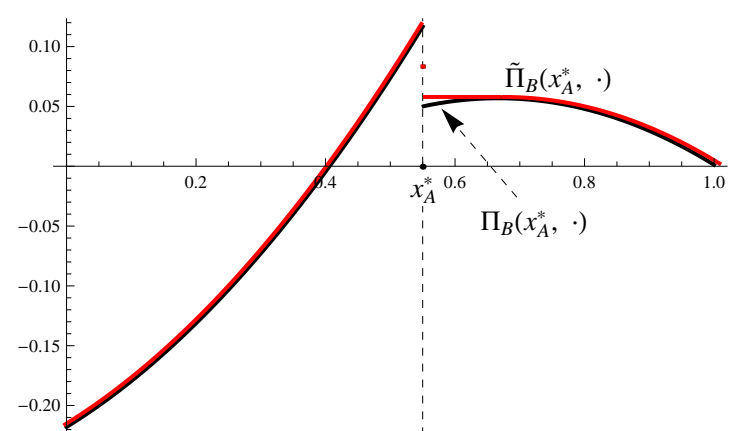

Figure 3: Quasi-concave hull $\tilde{\Pi}_{B}\left(x_{A}^{*}, \cdot\right)$ given $x_{A}^{*}=\frac{11}{20}$.

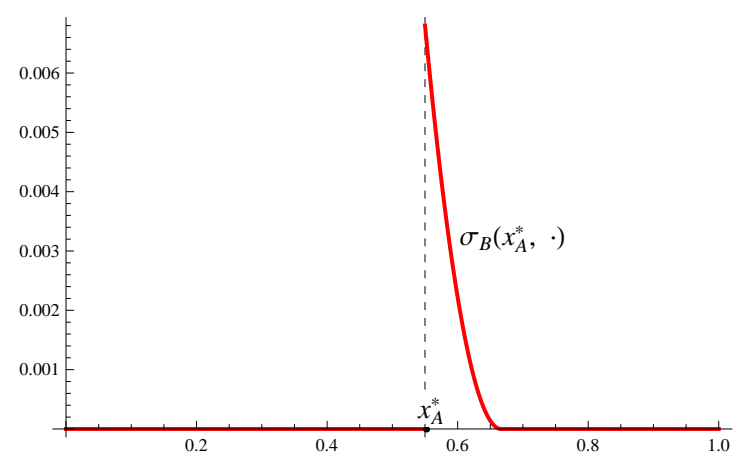

Figure 4: Index $\sigma_{B}$ of lack of quasiconcavity for $\Pi_{B}\left(x_{A}^{*}, \cdot\right)$ given $x_{A}^{*}=\frac{11}{20}$.

Let $d: \mathbb{R}^{k} \rightarrow \mathbb{R}_{+}$denote the Euclidean distance on $\mathbb{R}^{k}$, and let $B_{\delta}(y)=\left\{x \in \mathbb{R}^{k}\right.$ : $d(x, y)<\delta\}$ be the open ball about $y$ with radius $\delta>0$.

Definition 2 Party $i$ can secure a payoff $\alpha \in \mathbb{R}$ at $\left(x_{i}, x_{-i}\right) \in X \times X$ if there exists $\tilde{x}_{i} \in X$ and $\delta>0$ such that for all $x_{-i}^{\prime} \in B_{\delta}\left(x_{-i}\right), \Pi_{i}\left(\tilde{x}_{i}, x_{-i}^{\prime}\right) \geq \alpha$.

In words, party $i$ can secure a payoff $\alpha$ at $\left(x_{i}, x_{-i}\right) \in X \times X$ if it has a strategy that guarantees at least that payoff even if the other slightly deviates from $x_{-i}$.

Let $\Pi: X \times X \rightarrow \mathbb{R}^{2}$ be the vector payoff function of $\mathcal{G}$, defined as $\Pi(x)=$ $\left(\Pi_{A}(x), \Pi_{B}(x)\right)$ for all $x \in X \times X$. The graph of $\Pi$ is a subset of $X \times X \times \mathbb{R}^{2}$; specifically, $\operatorname{graph}(\Pi)=\left\{\left(x_{A}, x_{B}, \Pi_{A}, \Pi_{B}\right) \in X \times X \times \mathbb{R}^{2}: \forall i, \Pi_{i}=\Pi_{i}\left(x_{A}, x_{B}\right)\right\}$. Let $c l(\operatorname{graph}(\Pi))$ be the closure of the graph of the vector payoff function. ${ }^{10}$

Definition 3 The election game $\mathcal{G}=\left(X, \Pi_{i}\right)_{i=A, B}$ is said to be strongly better reply secure if for all $(\bar{x}, \bar{\Pi}) \in \operatorname{cl}(\operatorname{graph}(\Pi))$ such that $\bar{x}$ is not a pure strategy equilibrium, some party $i$ can secure a payoff strictly greater than $\bar{\Pi}_{i}+\sigma_{i}(\bar{x})$.

\footnotetext{
${ }^{10}$ Recall that $(\bar{x}, \bar{\Pi}) \in \operatorname{cl}(\operatorname{graph}(\Pi))$ if and only if for all $\epsilon>0, B_{\epsilon}(\bar{x}, \bar{\Pi}) \cap \operatorname{graph}(\Pi) \neq \emptyset$.
} 
Looking at Definition 3, it is clear that for compact and quasi-concave games, SBRS coincides with Reny's (1999) definition of better reply security, since in that case $\sigma_{i}(\bar{x})=0$ for all $i$ and all $\bar{x}$. In general, however, SBRS strengthens Reny's concept, since every strongly better reply secure game is better reply secure; but the class of compact and strongly better reply secure games strictly generalizes the class of compact, quasi-concave and better reply secure games (Prop 3.5, Bich 2009, p.401).

Example 3 (cont. Example 1) As was said before, the proportionally power sharing election game introduced in Section 2 is not always SBRS. To see this, consider again Example 1. First, note that the profile $\bar{x}=\left(\bar{x}_{A}, \bar{x}_{B}\right)$, with $\bar{x}_{A}=\bar{x}_{B}=0.509288$, is not a pure strategy equilibrium, since the conditional payoff function of $A$ given $\bar{x}_{B}$ (i.e., the dark curve of Fig. 5) jumps up discontinuously at $\bar{x}_{A}$ as $A$ moves his platform slightly to the left. Second, consider $(\bar{x}, \bar{\Pi}) \in \operatorname{cl}(\operatorname{graph}(\Pi))$, where $\bar{\Pi}_{A}=\limsup _{x_{A} \rightarrow \bar{x}_{A}} \Pi_{A}\left(x_{A}, \bar{x}_{B}\right)$ and $\bar{\Pi}_{B}=\liminf _{x_{B} \rightarrow \bar{x}_{B}} \Pi_{B}\left(\bar{x}_{A}, x_{B}\right)$. Since $\Pi_{B}\left(\bar{x}_{A}, \cdot\right)$ is not quasi-concave, $\sigma_{B}(\bar{x})>0$; actually $\sigma_{B}$ is determined by the distance between the red and the black curves of Fig. 6 , which represent the quasi-concave hull and the conditional payoff of $B$, respectively. Notice that $B$ cannot secure $\bar{\Pi}_{B}+\sigma_{B}(\bar{x})$, since as is illustrated by Fig. 6's dashed curve, $\Pi_{B}\left(\bar{x}_{A}-\delta, x_{B}^{\prime}\right)<\bar{\Pi}_{B}+\sigma_{B}(\bar{x})$ for any $\delta>0$ small enough and all $x_{B}^{\prime} \in X$. Following an analogous reasoning, Fig. 5 shows that party $A$ is unable to secure $\bar{\Pi}_{A}+\sigma_{A}(\bar{x})$ as well, though $\Pi_{A}\left(\cdot \bar{x}_{B}\right)$ is quasi-concave on $X$ and $\sigma_{A}(\bar{x})=0$. Thus, this election game is not strongly better reply secure.

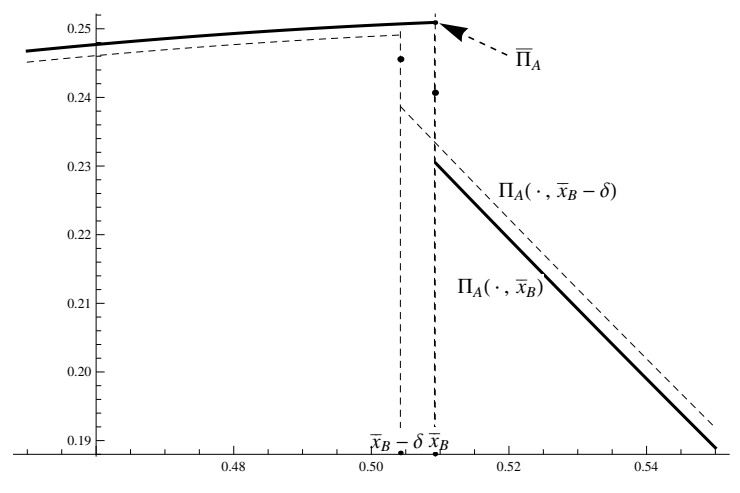

Figure 5: Party $A$ 's conditional payoff given $\bar{x}_{B}=0.509288\left(\right.$ resp. $\left.\bar{x}_{B}-\delta\right)$.

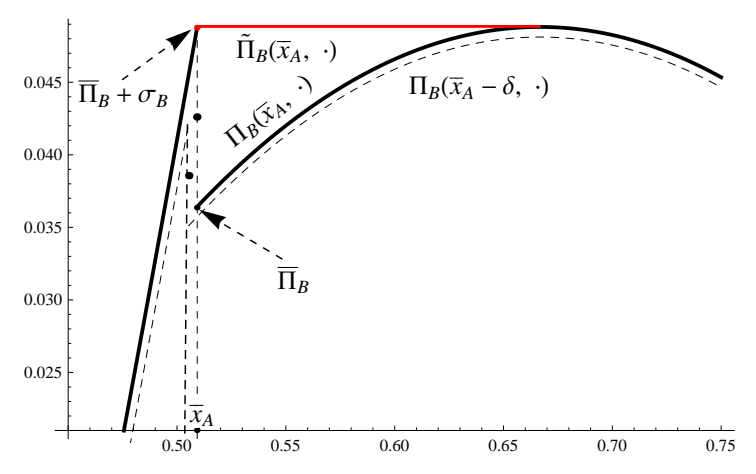

Figure 6: Party B's conditional payoff given $\bar{x}_{A}=0.509288\left(\right.$ resp. $\left.\bar{x}_{A}-\delta\right)$.

In the next pages, we define two conditions that imply together SBRS. These conditions are Reny's (1999) payoff security and a generalization of reciprocal upper semicontinuity for non quasi-concave frameworks proposed by Bich (2009).

Definition 4 The election game $\mathcal{G}=\left(X, \Pi_{i}\right)_{i=A, B}$ is said to be payoff secure if for all $x \in X \times X$ and all $\epsilon>0$, each party $i$ can secure a payoff of $\Pi_{i}(x)-\epsilon$ at $x$. 
Payoff security requires that for every profile $x$, each party has a strategy that virtually guarantees the payoff it receives at $x$ even if the other party slightly deviates from $x$.

Proposition 1 The election game $\mathcal{G}=\left(X, \Pi_{i}\right)_{i=A, B}$ is payoff secure.

The strategy behind the proof of Proposition 1 is to show that because of the discontinuities of the vote shares, when parties tie at any point on the diagonal of the strategy space $X \times X$ (except at the median type's preferred policy), there is always a suitable deviation off the diagonal that provides to the deviating party a discontinuous increase in the vote share that outweighs any utility loss from being farther away from the ideal policy. This argument carries over so long as there is positive weight assigned to vote motivation, regardless of how small such weight might be. ${ }^{11}$

Proof Suppose, by contradiction, there exists a strategy profile $\bar{x}=\left(\bar{x}_{A}, \bar{x}_{B}\right) \in X \times X$ and $\bar{\epsilon}>0$ such that for some $i$ the payoff $\Pi_{i}(\bar{x})-\bar{\epsilon}$ can't be secure at $\bar{x}$. This implies that for all $\tilde{x}_{i} \in X$ and all $\delta>0$, there exists $x_{-i}^{\prime} \in B_{\delta}\left(\bar{x}_{-i}\right)$ such that

$$
\Pi_{i}\left(\tilde{x}_{i}, x_{-i}^{\prime}\right)<\Pi_{i}\left(\bar{x}_{i}, \bar{x}_{-i}\right)
$$

By Lemma 3, if either (i) $\bar{x}_{i} \neq \bar{x}_{-i}$, (ii) $\bar{x}_{i}=\hat{x}\left(\theta_{M}\right)$, or (iii) $\lambda_{i}=0$, then $\Pi_{i}\left(\bar{x}_{i}, \cdot\right)$ is continuous in $x_{-i}$ at $\bar{x}_{-i}$. Moreover, by definition of continuity, there exists $\bar{\delta}>0$ such that $\Pi_{i}\left(\bar{x}_{i}, x_{-i}^{\prime}\right)>\Pi_{i}\left(\bar{x}_{i}, \bar{x}_{-i}\right)-\bar{\epsilon}$ for all $x_{-i}^{\prime} \in B_{\bar{\delta}}\left(\bar{x}_{-i}\right)$, contradicting that $i$ can't secure the payoff $\Pi_{i}(\bar{x})-\bar{\epsilon}$ at $\bar{x}$. Therefore, $\bar{x}_{i}=\bar{x}_{-i}=\bar{x} \neq \hat{x}\left(\theta_{M}\right)$ and $\lambda_{i}>0 .{ }^{12}$ Moreover,

$$
\Pi_{i}(\bar{x}, \bar{x})=\left(1-\lambda_{i}\right)\left(\frac{\lambda_{i}}{1-\lambda_{i}} \frac{1}{2}+U\left(\bar{x}, \theta_{i}\right)\right) .
$$

Without loss of generality, fix $i=A$. Two cases are possible. ${ }^{13}$

Case 1: $\hat{x}\left(\theta_{A}\right)<\bar{x}<\hat{x}\left(\theta_{M}\right)$. Consider $\tilde{x}_{A}>\bar{x}$ sufficiently closed to $\bar{x}$. Define $\bar{\delta}=$ $d\left(\bar{x}, \tilde{x}_{A}\right)$ and $B_{\bar{\delta}}(\bar{x})$. Choose $\tilde{x}_{A}$ in such a way that $\bar{x}-\bar{\delta}>\hat{x}\left(\theta_{A}\right)$ and $\bar{x}+\bar{\delta}<\hat{x}\left(\theta_{M}\right)$. Since $\hat{x}\left(\theta_{A}\right)<\bar{x}<\hat{x}\left(\theta_{M}\right)$, this number always exists. For any $x_{B}^{\prime} \in B_{\bar{\delta}}(\bar{x})$, let $x_{B}^{\prime}=\bar{x}+\alpha \bar{\delta}$, with $\alpha \in(-1,1)$, and recall $\tilde{x}_{A}=\bar{x}+\bar{\delta}$. Then, $x\left(\tilde{x}_{A}, x_{B}^{\prime}\right)=\bar{x}+\bar{\delta}\left[\rho_{A}\left(\tilde{x}_{A}, x_{B}^{\prime}\right)(1-\alpha)+\alpha\right]$; and from (10)

$$
\begin{aligned}
& \Pi_{A}\left(\tilde{x}_{A}, x_{B}^{\prime}\right)-\Pi_{A}(\bar{x}, \bar{x})= \\
& \quad\left(1-\lambda_{A}\right)\left(\frac{\lambda_{A}}{1-\lambda_{A}}\left(\rho_{A}\left(\tilde{x}_{A}, x_{B}^{\prime}\right)-\frac{1}{2}\right)+U\left(x\left(\tilde{x}_{A}, x_{B}^{\prime}\right), \theta_{A}\right)-U\left(\bar{x}, \theta_{A}\right)\right) .
\end{aligned}
$$

\footnotetext{
${ }^{11}$ Off the diagonal (resp., at the profile of median preferred policies), payoff security follows from the continuity of the payoffs. The same happens when $\lambda_{i}=0$ for all $i$.

${ }^{12}$ In an abuse of notation, in the rest of this proof $\bar{x}$ is employed at the same time to denote the vector of identical platforms and the corresponding common value.

${ }^{13}$ The reader should verify that the remaining possibility, i.e. $\bar{x} \leq \hat{x}\left(\theta_{A}\right)$, is a variation of these cases.
} 
On the one hand, note that by Lemma $1, \rho_{A}\left(\tilde{x}_{A}, x_{B}^{\prime}\right)=F\left(\left[\bar{\theta}\left(\tilde{x}_{A}, x_{B}^{\prime}\right), 1\right]\right)>\frac{1}{2}$ because $\bar{\theta}\left(\tilde{x}_{A}, x_{B}^{\prime}\right)<\theta_{M}$. In fact, no matter how small we define $\bar{\delta}$, party $A$ 's share of votes $\rho_{A}\left(\tilde{x}_{A}, \bar{x}\right)$ jumps up discontinuously above $1 / 2$ as $x_{A}$ moves away from $\bar{x}$ towards $\tilde{x}_{A}=$ $\bar{x}+\bar{\delta}$, and it remains well above $1 / 2$ for any $x_{B}^{\prime}$ due to the continuity of $\rho_{A}(\cdot)$ for $x_{A} \neq x_{B}$. On the other hand, given that $\bar{\delta}\left[\rho\left(\tilde{x}_{A}, x_{B}^{\prime}\right)(1-\alpha)+\alpha\right]>0$, the term $U\left(x\left(\tilde{x}_{A}, x_{B}^{\prime}\right), \theta_{A}\right)-$ $U\left(\bar{x}, \theta_{A}\right)$ is negative; but by Ass 2 it converges to zero as $\bar{\delta} \rightarrow 0$. Therefore, for $\bar{\delta}$ small enough (i.e., for $\tilde{x}_{A}$ sufficiently closed to $\bar{x}$ ), we have that $\Pi_{A}\left(\tilde{x}_{A}, x_{B}^{\prime}\right)-\Pi_{A}(\bar{x}, \bar{x})>0$ for all $x_{B}^{\prime} \in B_{\bar{\delta}}(\bar{x})$, providing the desired contradiction with (9).

Case 2: $\hat{x}\left(\theta_{M}\right)<\bar{x}$. Consider $\tilde{x}_{A}<\bar{x}$ and $\bar{\delta}=d\left(\bar{x}, \tilde{x}_{A}\right)$, such that $\tilde{x}_{A}=\bar{x}-\bar{\delta}>\hat{x}\left(\theta_{M}\right)$. For any $x_{B}^{\prime} \in B_{\bar{\delta}}(\bar{x})$, we have that $\rho\left(\tilde{x}_{A}, x_{B}^{\prime}\right)=F\left(\left[0, \bar{\theta}\left(\tilde{x}_{A}, x_{B}^{\prime}\right)\right]\right)$ and that $x\left(\tilde{x}_{A}, x_{B}^{\prime}\right)=$ $\bar{x}+\bar{\delta}\left[\alpha-\rho\left(\tilde{x}_{A}, x_{B}^{\prime}\right)(1+\alpha)\right]$, where $\alpha \in(-1,1)$. Thus, given that $\bar{\theta}\left(\tilde{x}_{A}, x_{B}^{\prime}\right)>\theta_{M}$, it follows that $\rho\left(\tilde{x}_{A}, x_{B}^{\prime}\right)>1 / 2$ and, consequently, that $x\left(\tilde{x}_{A}, x_{B}^{\prime}\right)<\bar{x}$. Moreover, by Ass 2 , $U\left(x\left(\tilde{x}_{A}, x_{B}^{\prime}\right), \theta_{A}\right)$ is greater than $U\left(\bar{x}, \theta_{A}\right)$. Hence, from $(11), \Pi_{A}\left(\tilde{x}_{A}, x_{B}^{\prime}\right)>\Pi_{A}(\bar{x}, \bar{x})$ for all $x_{B}^{\prime} \in B_{\bar{\delta}}(\bar{x})$, contradicting again (9).

Now we introduce the second condition, called weakly reciprocally upper semicontinuity (WRUSC).

Definition 5 The election game $\mathcal{G}=\left(X, \Pi_{i}\right)_{i=A, B}$ is said to be weakly reciprocally upper semi-continuous if for all $(\bar{x}, \bar{\Pi}) \in \operatorname{cl}(\operatorname{graph}(\Pi)) \backslash \operatorname{graph}(\Pi-\sigma)$, there is a party $i$ and a strategy $\tilde{x}_{i} \in X$ for $i$ such that $\Pi_{i}\left(\tilde{x}_{i}, \bar{x}_{-i}\right)>\bar{\Pi}_{i}+\sigma_{i}(\bar{x})$.

Contrary to what happens with payoff security, Example 3 illustrates that for arbitrary values of $\lambda_{i} \in(0,1)$, the proportionally power sharing election game does not possess the property of being weakly reciprocally upper semi-continuous. Indeed, given that $\mathcal{G}$ is always payoff secure (Proposition 1), its failure to be strongly better reply secure (and to possess a Nash equilibrium in pure strategies) for the parameter values of Example 3 must be attributed to the fact that $\mathcal{G}$ violates WRUSC. ${ }^{14}$ That's exactly where the root of the electoral instability lies; and, as we argue below, it is a direct consequence of the heterogeneity of the political motivations (Proposition 2).

To elaborate, Proposition 1 indicates that in consensual democracies the discontinuities of the conditional payoffs do not preclude the contesting parties "to protect" their payoffs from the undercutting behavior of their rivals. The trouble, however, is that when such behavior takes place at any point on the diagonal, although the payoffs jump in opposite directions, the shift of the electorate from one party to the other does not posses the same utility value for the parties when these are differently concerned with

\footnotetext{
${ }^{14}$ Bear in mind that payoff security together with WRUSC imply SBRS (Bich 2009).
} 
power. For instance, given that in Example 3 party $A$ assigns more weight on the vote share than $B$, the utility loss due to a reduction of A's percentage of votes is bigger than the utility gain due to a similar increase in $B$ 's vote share. As a result, the sum of the payoffs $\sum_{i} \Pi_{i}(\cdot)$ is neither lower nor upper semi-continuous. The following lemma shows that this never occur when political parties share the same electoral motivations.

Lemma 4 If $\lambda_{i}=\lambda \in[0,1]$ for all $i=A, B$, then the sum of the payoff functions $\sum_{i} \Pi_{i}\left(x_{A}, x_{B}\right)$ associated to the election game $\mathcal{G}=\left(X, \Pi_{i}\right)_{i=A, B}$ is continuous on $X \times X$.

Proof Under the hypothesis of the current lemma, from (5) and (6) we have that for every strategy profile $\left(x_{A}, x_{B}\right) \in X \times X$,

$$
\sum_{i} \Pi_{i}\left(x_{A}, x_{B}\right)=\lambda+(1-\lambda)\left(U_{A}\left(x\left(x_{A}, x_{B}\right)\right)+U_{B}\left(x\left(x_{A}, x_{B}\right)\right)\right),
$$

where $x\left(x_{A}, x_{B}\right)$ is given by (1).

Suppose first that $x_{A} \neq x_{B}$. By Lemma $1, \rho_{i}\left(x_{A}, x_{B}\right)=F\left(\left[0, \bar{\theta}\left(x_{A}, x_{B}\right)\right]\right)$ and $\rho_{-i}\left(x_{A}, x_{B}\right)=F\left(\left[\bar{\theta}\left(x_{A}, x_{B}\right), 1\right]\right)$ for some $i=A, B$. By Ass 1 and Ass $5, \rho_{i}(\cdot)$ and $\rho_{-i}(\cdot)$ are both continuous at $\left(x_{A}, x_{B}\right)$; and by definition so is the implemented policy $x(\cdot)$. Therefore, the continuity of $\sum_{i} \Pi_{i}(\cdot)$ at $\left(x_{A}, x_{B}\right)$ follows from Ass 2, i.e., by the continuity of the utility function $U_{i}(\cdot) \equiv U\left(\cdot, \theta_{i}\right)$.

Alternatively, if $x_{A}=x_{B}\left(=x^{\prime} \in X\right)$, then by hypothesis $\rho_{i}\left(x^{\prime}, x^{\prime}\right)=1 / 2$, and

$$
\sum_{i} \Pi_{i}\left(x^{\prime}, x^{\prime}\right)=\lambda+(1-\lambda)\left(U\left(x^{\prime}, \theta_{A}\right)+U\left(x^{\prime}, \theta_{B}\right)\right) .
$$

The rest of the proof follows the argument of the proof of Lemma 3. Specifically, consider a sequence $x^{n}=\left(x_{A}^{n}, x_{B}^{n}\right) \subseteq X \times X$ such that $\left(x_{A}^{n}, x_{B}^{n}\right) \rightarrow\left(x^{\prime}, x^{\prime}\right)$. Note that $\lim _{x^{n} \rightarrow x^{\prime}} x\left(x_{A}^{n}, x_{B}^{n}\right)=x^{\prime}$. Therefore, by Ass 2 and the expressions in (12) and (13), it follows that $\sum_{i} \Pi_{i}\left(x^{n}\right) \rightarrow \sum_{i} \Pi_{i}\left(x^{\prime}\right)$ as $x^{n} \rightarrow x^{\prime}$. This completes the proof.

We now employ Lemma 4 to show that when both parties exhibit the same electoral interests, consensual democracies satisfy weakly reciprocally upper semi-continuity.

Proposition 2 If $\lambda_{i}=\lambda \in[0,1]$ for all $i=A, B$, then $\mathcal{G}=\left(X, \Pi_{i}\right)_{i=A, B}$ is weakly reciprocally upper semi-continuous.

Proof Consider any element $(\bar{x}, \bar{\Pi})$ of $c l(\operatorname{graph}(\Pi)) \backslash \operatorname{graph}(\Pi-\sigma)$. If $\sigma(\bar{x})=0$, then $(\bar{x}, \bar{\Pi})$ belongs to the closure of the graph of $\Pi$ but not to the graph; i.e., $(\bar{x}, \bar{\Pi}) \in$ $c l(\operatorname{graph}(\Pi)) \backslash \operatorname{graph}(\Pi)$. Thus $\bar{\Pi}_{i} \neq \Pi_{i}(\bar{x})$ for some $i$. If $\bar{\Pi}_{i}<\Pi_{i}(\bar{x})$ we are done. 
Otherwise, if $\bar{\Pi}_{i}>\Pi_{i}(\bar{x})$, then $\bar{\Pi}_{-i}<\Pi_{-i}(\bar{x})$, because by Lemma 4 we know that $\bar{\Pi}_{i}+\bar{\Pi}_{-i}=\Pi_{i}(\bar{x})+\Pi_{-i}(\bar{x})$.

On the other hand, if $\sigma(\bar{x}) \neq 0$, then we proceed as follows. Suppose without loss of generality that $\sigma_{i}(\bar{x})>0$. Thus $\sigma_{i}(\bar{x})+\sigma_{-i}(\bar{x})>0$; and using the definition of $\sigma$,

$$
\limsup _{x_{i} \rightarrow \bar{x}_{i}} \tilde{\Pi}_{i}\left(x_{i}, \bar{x}_{-i}\right)+\limsup _{x_{-i} \rightarrow \bar{x}_{-i}} \tilde{\Pi}_{-i}\left(\bar{x}_{i}, x_{-i}\right)>\underbrace{\limsup _{x \rightarrow \bar{x}}\left[\Pi_{i}\left(x_{i}, \bar{x}_{-i}\right)+\Pi_{-i}\left(\bar{x}_{i}, x_{-i}\right)\right]}_{=\bar{\Pi}_{i}+\bar{\Pi}_{-i} \text { by Lemma } 4} .
$$

Therefore,

$$
\limsup _{x_{i} \rightarrow \bar{x}_{i}} \tilde{\Pi}_{i}\left(x_{i}, \bar{x}_{-i}\right)+\limsup _{x_{-i} \rightarrow \bar{x}_{-i}} \tilde{\Pi}_{-i}\left(\bar{x}_{i}, x_{-i}\right)>\bar{\Pi}_{i}+\bar{\Pi}_{-i}
$$

Suppose $\bar{x}_{i}=\bar{x}_{-i}$. (The other case with $\bar{x}_{i} \neq \bar{x}_{-i}$ is similar). Note that $\sigma_{i}(\bar{x})>0$ implies $\Pi_{i}\left(\cdot, \bar{x}_{-i}\right)$ jumps down at $\bar{x}_{i}$. First, if $\lim \sup _{x_{i} \rightarrow \bar{x}_{i}} \tilde{\Pi}_{i}\left(x_{i}, \bar{x}_{-i}\right)=\bar{\Pi}_{i}$, then from (14), $\lim \sup _{x_{-i} \rightarrow \bar{x}_{-i}} \tilde{\Pi}_{-i}\left(\bar{x}_{i}, x_{-i}\right)>\bar{\Pi}_{-i}$. Moreover, by Lemma 4, $\Pi_{-i}\left(\bar{x}_{i}, \cdot\right)$ jumps up at $\bar{x}_{-i}$; and by assumptions Ass 1-6, $\sigma_{-i}(\bar{x})=0$ and $\lim \sup _{x_{-i} \rightarrow \bar{x}_{-i}} \tilde{\Pi}_{-i}\left(\bar{x}_{i}, x_{-i}\right)=$ $\lim \sup _{x_{-i} \rightarrow \bar{x}_{-i}} \Pi_{-i}\left(\bar{x}_{i}, x_{-i}\right)$. Therefore, limsup $\sup _{x_{-i} \rightarrow \bar{x}_{-i}} \Pi_{-i}\left(\bar{x}_{i}, x_{-i}\right)>\bar{\Pi}_{-i}$; and by continuity of $\Pi_{-i}\left(\bar{x}_{i}, \cdot\right)$ for $x_{-i} \neq \bar{x}_{-i}$, there must exists $\tilde{x}_{-i} \in X$ closed enough to $\bar{x}_{-i}$ such that $\Pi_{-i}\left(\bar{x}_{i}, \tilde{x}_{-i}\right)>\bar{\Pi}_{-i}$.

Second, if $\lim \sup _{x_{i} \rightarrow \bar{x}_{-i}} \Pi_{i}\left(x_{i}, \bar{x}_{-i}\right)>\bar{\Pi}_{i}+\sigma_{i}(\bar{x})$, as is illustrated in Fig. 7a, then we use the argument of continuity employed above. To be concrete, since $\Pi_{i}\left(\cdot, \bar{x}_{-i}\right)$ is continuous for $x_{i} \neq \bar{x}_{-i}$, there exists $\tilde{x}_{i} \in X$ closed enough to $\bar{x}_{i}$ such that $\Pi_{i}\left(\tilde{x}_{i}, \bar{x}_{-i}\right)>\bar{\Pi}_{i}+\sigma_{i}(\bar{x})$.

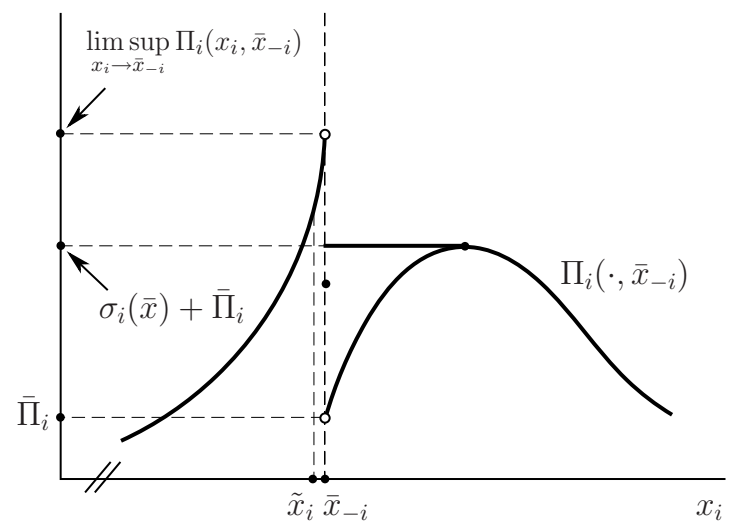

(a) $\lim \sup _{x_{i} \rightarrow \bar{x}_{-i}} \Pi_{i}\left(x_{i}, \bar{x}_{-i}\right)>\bar{\Pi}_{i}+\sigma_{i}(\bar{x})$.

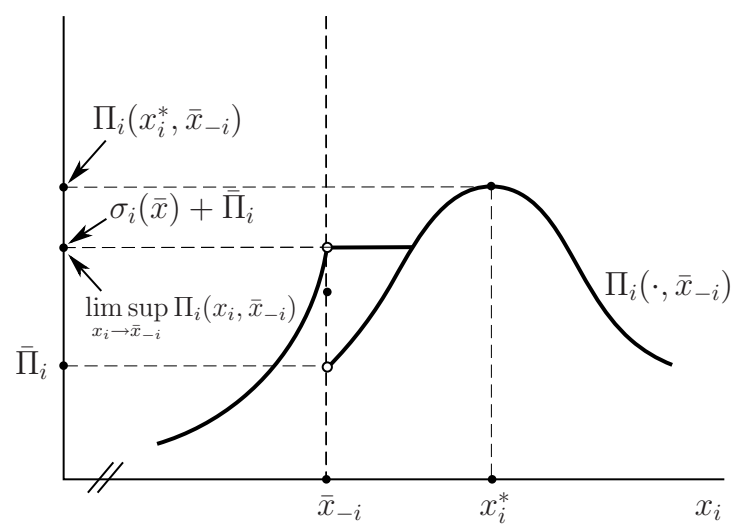

(b) $\lim \sup _{x_{i} \rightarrow \bar{x}_{-i}} \Pi_{i}\left(x_{i}, \bar{x}_{-i}\right)=\bar{\Pi}_{i}+\sigma_{i}(\bar{x})$.

Figure 7: Proof of Proposition 2.

Finally, the last possibility illustrated in Fig. 7b takes place when

$$
\limsup _{x_{i} \rightarrow \bar{x}_{-i}} \Pi_{i}\left(x_{i}, \bar{x}_{-i}\right)=\bar{\Pi}_{i}+\sigma_{i}(\bar{x})
$$


Let $x_{i}^{*} \equiv \arg \max _{x_{i}} \tilde{\Pi}_{i}\left(x_{i}, \bar{x}_{-i}\right)$. Recall that $x_{i}^{*}$ exists because $\tilde{\Pi}_{i}\left(\cdot, \bar{x}_{-i}\right)$ is quasi-concave; and that it is unique by Ass 1-6. Clearly, $\tilde{\Pi}_{i}\left(x_{i}^{*}, \bar{x}_{-i}\right)>\lim \sup _{x_{i} \rightarrow \bar{x}_{-i}} \tilde{\Pi}_{i}\left(x_{i}, \bar{x}_{-i}\right)=$ $\lim \sup _{x_{i} \rightarrow \bar{x}_{-i}} \Pi_{i}\left(x_{i}, \bar{x}_{-i}\right)$, where the last equality follows from the quasi-concavity of $\Pi_{i}\left(\cdot, \bar{x}_{-i}\right)$ at $\bar{x}_{-i}$. (Otherwise, if $\tilde{\Pi}_{i}\left(x_{i}^{*}, \bar{x}_{-i}\right)<\lim \sup _{x_{i} \rightarrow \bar{x}_{-i}} \tilde{\Pi}_{i}\left(x_{i}, \bar{x}_{-i}\right)$, then $\sigma_{i}(\bar{x})$ would have been smaller than $\lim \sup _{x_{i} \rightarrow \bar{x}_{-i}} \Pi_{i}\left(x_{i}, \bar{x}_{-i}\right)-\bar{\Pi}_{i}$, which would contradict (15)). Moreover, (15) implies that $\tilde{\Pi}_{i}\left(x_{i}^{*}, \bar{x}_{-i}\right)=\Pi_{i}\left(x_{i}^{*}, \bar{x}_{-i}\right)$, because $\Pi_{i}\left(x_{i}, \bar{x}_{-i}\right)$ is single-peaked for $x_{i}>\bar{x}_{i}$. Therefore, $\Pi_{i}\left(x_{i}^{*}, \bar{x}_{-i}\right)>\bar{\Pi}_{i}+\sigma_{i}(\bar{x})$.

Finally, we state and prove the main result of the paper.

Theorem 1 If $\lambda_{i}=\lambda \in[0,1]$ for all $i=A, B$, then the proportionally power sharing election game $\mathcal{G}=\left(X, \Pi_{i}\right)_{i=A, B}$ possesses a pure strategy equilibrium.

Proof First, note that $\mathcal{G}$ is compact because (i) $X$ is a nonempty, compact and convex subset of the real line, and (ii) $\Pi_{i}$ is bounded (both $\rho_{i}(\cdot)$ and $U_{i}(\cdot)$ are bounded). Second, by Propositions 1 and $2, \mathcal{G}$ is payoff secure and reciprocally upper semi-continuous. Therefore, $\mathcal{G}$ is strongly better reply secure (Bich 2009, Proposition 4.4); and thereby it possesses a pure strategy equilibrium (Bich 2009, Theorem 3.2) ${ }^{15}$

Consider the election game discussed in Example 1, which is a workhorse model of electoral competition, and suppose the two parties possess symmetric motives, in the sense that for all $i=A, B, \lambda_{i}=\lambda$ for some $\lambda \in(0,1)$. Then, the equilibrium policies as a function of this common value of $\lambda$ are as follows.

Proposition 3 Suppose $\theta$ is uniformly distributed on $[0,1]$ and let $U(x, \theta)=-\|x-\theta\|$. Then, if $\lambda_{i}=\lambda$ for all $i=A, B$, the election game $\mathcal{G}=\left(X, \pi_{i}\right)_{i=A, B}$ has a unique pure strategy equilibrium with the property that:

1. If $\lambda<1 / 2$, then $x_{A}^{*}(\lambda)=\frac{\lambda}{2(1-\lambda)}<\frac{1}{2}<1-\frac{\lambda}{2(1-\lambda)}=x_{B}^{*}(\lambda)$;

2. If $\lambda \geq 1 / 2$, then $x_{A}^{*}(\lambda)=\frac{1}{2}=x_{B}^{*}(\lambda)$;

3. For all $\lambda \in[0,1), \rho_{i}\left(x_{A}^{*}(\lambda), x_{B}^{*}(\lambda)\right)=1 / 2$;

4. For all $\lambda \in[0,1), x\left(x_{A}^{*}(\lambda), x_{B}^{*}(\lambda)\right)=1 / 2$.

Proof Under the hypotheses of Proposition 3, a Nash equilibrium in pure strategies $\left(x_{A}^{*}, x_{B}^{*}\right)$ always exists. Moreover, it is easy to verify that $x_{A}^{*} \leq x_{B}^{*}$ and that $\theta_{A}<$ $x\left(x_{A}^{*}, x_{B}^{*}\right)<\theta_{B}$. Suppose first $x_{A}^{*}<x_{B}^{*}$. Since $\rho_{i}(\cdot)$ is continuous for $x_{A} \neq x_{B}$, then

\footnotetext{
${ }^{15}$ Note that showing that $\mathcal{G}$ is better reply secure is not enough because even after restricting $\lambda_{i}$ to be equal for both parties, the conditional payoff functions need not be quasi-concave.
} 
must exist $\epsilon>0$ sufficiently small such that for all $\left(x_{A}, x_{B}\right) \in X_{\epsilon}\left(x_{A}^{*}\right) \times X_{\epsilon}\left(x_{B}^{*}\right), \theta_{A}<$ $x\left(x_{A}, x_{B}\right)<\theta_{B}$, where $X_{\epsilon}\left(x_{i}^{*}\right)=\left(x_{i}^{*}-\epsilon, x_{i}^{*}+\epsilon\right), i=A, B$. Thus, for any strategy profile $\left(x_{A}, x_{B}\right) \in X_{\epsilon}\left(x_{A}^{*}\right) \times X_{\epsilon}\left(x_{B}^{*}\right)$, party $A$ 's payoff function can be written as

$$
\Pi_{A}\left(x_{A}, x_{B}\right)=\lambda\left(\frac{x_{A}+x_{B}}{2}\right)-(1-\lambda)\left[\left(\frac{x_{A}+x_{B}}{2}\right) x_{A}+\left(1-\frac{x_{A}+x_{B}}{2}\right) x_{B}-\theta_{A}\right] .
$$

Fix $x_{B}^{*} \in X_{\epsilon}\left(x_{B}^{*}\right)$, and consider party $A$ 's best response to $x_{B}^{*}$ over $X_{\epsilon}\left(x_{A}^{*}\right)$. This is obtained by solving the optimization problem $\max _{x_{A} \in X_{\epsilon}\left(x_{A}^{*}\right)} \Pi_{A}\left(x_{A}, x_{B}^{*}\right)$. The first order condition for this problem provides a stationary point $\frac{\lambda}{2(1-\lambda)}$. Note that this critical point actually maximizes the conditional payoff $\Pi_{A}\left(x_{A}, x_{B}^{*}\right)$ over $X_{\epsilon}\left(x_{A}^{*}\right)$ because $\Pi_{A}\left(x_{A}, x_{B}^{*}\right)$ has an interior maximum on $X_{\epsilon}\left(x_{A}^{*}\right) \cdot{ }^{16}$ Moreover, since $\Pi_{A}\left(\cdot, x_{B}^{*}\right)$ is strictly concave over $X_{\epsilon}\left(x_{A}^{*}\right)$, with $\frac{\partial^{2} \Pi_{A}\left(x_{A}, x_{B}^{*}\right)}{\partial x_{A}^{2}}=-(1-\lambda)<0$, the maximum is unique; and consequently $x_{A}^{*}=\frac{\lambda}{2(1-\lambda)}$. A similar argument shows that $x_{B}^{*}=1-\frac{\lambda}{2(1-\lambda)}$. Thus, $\rho_{i}\left(x_{A}^{*}, x_{B}^{*}\right)=1 / 2$ and $x\left(x_{A}^{*}, x_{B}^{*}\right)=1 / 2$. Finally, notice that $x_{A}^{*}<x_{B}^{*}$ if and only if $\lambda \leq 1 / 2$, which implies that $x_{A}^{*}<1 / 2<x_{B}^{*}$.

Let's now consider the possibility of a PSE where both parties propose the median type's preferred policy, i.e., where $x_{A}^{*}=x_{B}^{*}=1 / 2$. Consider first a deviation for party $A$ to any platform $x_{A}^{\prime}<1 / 2$. For convenience, let's write $x_{A}^{\prime}=1 / 2-\delta$, with $\delta>0$. Routine calculations show that $\Pi_{A}\left(x_{A}^{\prime}, x_{B}^{*}\right) \equiv 1 / 2\left(-1+\delta-2 \theta_{A}(-1+\lambda)+\delta^{2}(-1+\lambda)+2 \lambda-2 \delta \lambda\right)>$ $-1 / 2+\theta_{A}+\lambda-\theta_{A} \lambda \equiv \Pi_{A}\left(x_{A}^{*}, x_{B}^{*}\right)$ if and only if $\lambda<\frac{-1+\delta}{-2+\delta}$, which converges to $1 / 2$ as $\delta$ goes to zero. Thus, $A$ has no profitable deviations below the median if $\lambda \geq 1 / 2$. Second, imagine party $B$ deviates to any platform $x_{B}^{\prime}=1 / 2+\delta>1 / 2$. Repeating the previous argument, $\Pi_{B}\left(x_{A}^{*}, x_{B}^{\prime}\right) \equiv 1 / 2\left(1+\delta+2 \theta_{B}(-1+\lambda)+\delta^{2}(-1+\lambda)-2 \delta \lambda\right)>$ $1 / 2+\theta_{B}(-1+\lambda) \equiv \Pi_{B}\left(x_{A}^{*}, x_{B}^{*}\right)$ if and only if $\lambda<\frac{-1+\delta}{-2+\delta}$, which once again converges to $1 / 2$ as $\delta$ goes to zero. Thus, $B$ has no profitable deviations above the median if $\lambda \geq 1 / 2$. The careful reader should verify at this point that party $A$ (resp., $B$ ) has a profitable deviation above (resp. below) the median preferred policy if $\lambda<\frac{-1+\delta}{\delta}$, which converges to $-\infty$ as $\delta$ goes to zero and to -1 as $\delta$ goes to $1 / 2$. Hence, so long as $\lambda \geq 1 / 2$, the strategy profile $\left(x_{A}^{*}, x_{B}^{*}\right)=(1 / 2,1 / 2)$ constitutes a PSE for $\mathcal{G}$. Obviously, in this case $\rho_{i}(1 / 2,1 / 2)=1 / 2$ for all $i$, and $x=1 / 2$. Routine calculations also show that there is no other equilibrium in pure strategies with identical policies.

Figure 8 illustrates the equilibrium platforms characterized in Proposition 3. The graph shows in the green region how parties trade off ideology for power (votes) as their common relative interest in power $\lambda$ changes. In the purely ideological case with $\lambda=0$, each party campaigns on its own ideological side proposing the most radical platform

\footnotetext{
${ }^{16}$ Bear in mind that the assumption that $\left(x_{A}^{*}, x_{B}^{*}\right)$ is a PSE implies that $\Pi_{A}\left(x_{A}^{*}, x_{B}^{*}\right) \geq \Pi_{A}\left(x_{A}, x_{B}^{*}\right)$ for all $x_{A}^{*} \in X_{\epsilon}\left(x_{A}^{*}\right)$; i.e., $x_{A}^{*}$ is an interior maximizer of $\Pi_{A}\left(\cdot, x_{B}^{*}\right)$ on $X_{\epsilon}\left(x_{A}^{*}\right)$.
} 
possible. Parties become more moderate as their relative interest for power increases, moving their electoral policies towards the center in accordance with the stationary points of the first order conditions (which capture the costs and benefits of being more moderate). The median voter result is recovered when $\lambda$ achieves the critical value of $1 / 2$, which denotes an equal concern for both goals, policy and votes. From that point on, the electoral equilibrium of proportionally power sharing election games coincides with the equilibrium of winner-take-all electoral competition.

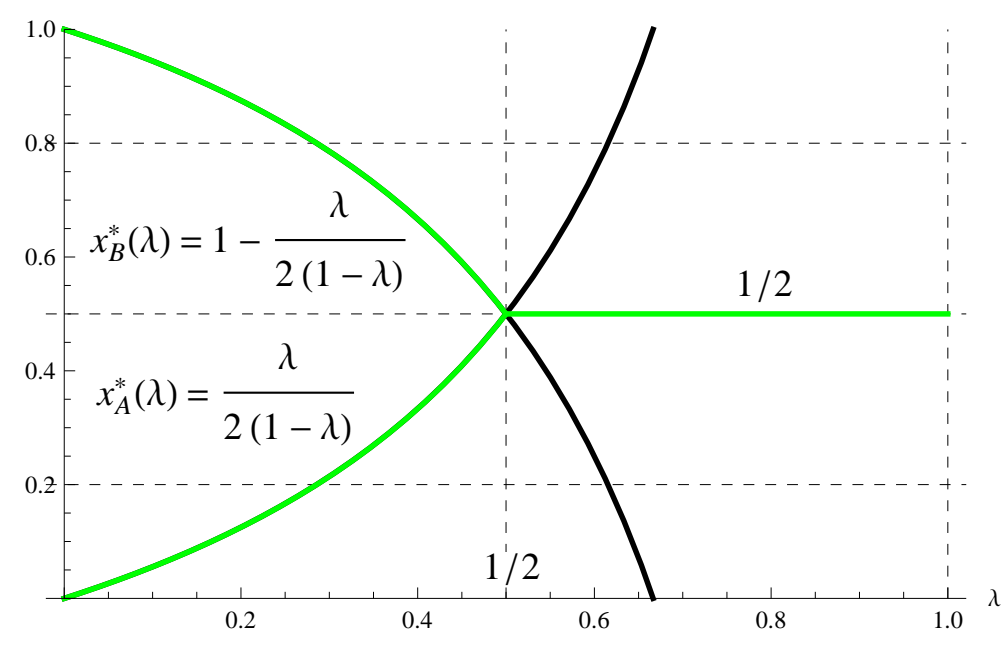

Figure 8: $\lambda_{A}=\lambda_{B}=\lambda$.

\section{Discussion}

This paper analyzes a model of democracy where political parties compete for policy influence in an election and afterwards they share power proportionally to the votes gathered in the contest. The paper uncovers the difficulties to guarantee the existence of a stable outcome in these polities, and it provides a sensible condition on political motivations that ensures the existence of pure strategy equilibrium. The main lesson is that, paradoxically, consensual democracies lead to more radical electoral campaigns. Indeed, the electoral equilibrium when it exists is shown to vary with the relative interest in power, starting with radical policy platforms when parties are solely concerned with ideology, and smoothly converging to the center of the policy space as parties get more appetite for power. Although electoral politics is as we explained considerably affected by parties' ideologies and their electoral motivations, the analysis shows that post-electoral politics responds exclusively to the median voter's preferences.

Despite the fact that the model considered here is rich enough to pick up several features of electoral competition that have been overlooked in the literature, there are 
a number of issues that would require more attention in future work. Among them, it would be interesting to relax the assumption of deterministic voting and to explore the effect of electoral uncertainty about the median voter's preferences. One way of doing this would be by permitting endogenous voter participation. Herrera et al. (2012) has recently studied a related problem in a Poisson game with population uncertainty, but they don't look at the effect of political motivations over voter turnout. Do electoral motivations and party ideology have any effects on voter participation? To put it differently, do office motivated candidates generate less enthusiasm in the electorate and higher abstention rates? Under which power sharing mechanism are these effects stronger?

In addition to the electoral uncertainty, there is at least another (more technical) issue that might be also worth exploring. The analysis in this paper focuses on the equilibria of a specific type of consensual democracy, in which the power sharing function (i.e. the influence on policy) is linear in parties' vote shares. But, of course, this function should depend on institutional and cultural factors. In general, the power sharing mechanism should be a nondecreasing function of the vote shares, but not necessarily a proportional or linear one. It could be even a discontinuous function if, for instance, going from $50-\epsilon \%$ to $50+\epsilon \%$ of the votes matters more in terms of policy making influence than going from say 80 to $82 \%$. What are the conditions for equilibrium existence in this more general model of consensual democracy? Does the symmetry of the electoral interests still ensure that the electoral game satisfy strongly better reply security? What's the effect of ideology over the equilibrium platforms and the implemented policy? We conjecture that the results found in this paper hold under a more general class of power sharing functions. However, a full analysis of this conjecture and a complete equilibrium characterization under different conditions of preferences and uncertainty are beyond the scope of this paper and they are left for future studies.

\section{References}

Bagnoli, M., and T. Bergstrom (2005) Log-concave probability and its applications, Economic Theory 26, 445-469.

Ball, R. (1999) Discontinuity and nonexistence of equilibrium in the probabilistic spatial voting model, Social Choice and Welfare, 16, 533-555.

Bernhardt, D., Duggan, J., and F. Squintani (2009) The case for responsible parties, American Political Science Review, 103, 570-587.

Bich, P. (2009) Existence of pure Nash equilibria in discontinuous and non quasiconcave games, International Journal of Game Theory, 38, 395-410. 
Callender, S. (2008) Political motivations, Review of Economic Studies, 75, 671-697.

Drouvelis, M., Saporiti, A., and N. Vriend (2011) Political motivations and electoral competition: Equilibrium analysis and experimental evidence, Economics Discussion Paper No. 1119, University of Manchester.

Duggan, J. (2012) A survey of equilibrium analysis in spatial models of elections, manuscript available at http://johnduggan.net.

Herrera, H., Morelli, M., and T. Palfrey (2012) Turnout and power sharing, mimeo.

Lijphart A. (1984) Democracies: patterns of majoritarian and consensus government in twenty-one countries. New Haven: Yale University Press.

Lizzeri, A., and N. Persico (2001) The provision of public goods under alternative electoral incentives, American Economic Review, 91, 225-239.

Persico, N., and N. Sahuguet (2006) Campaign spending regulation in a model of redistributive politics, Economic Theory, 28, 95-124.

Ortuño Ortín, I. (1997) A spatial model of political competition and proportional representation, Social Choice and Welfare 14, 427-238.

Reny, P. (1999) On the existence of pure and mixed strategy Nash equilibria in discontinuous games, Econometrica, 67, 1029-1056.

Roemer, J. (2001) Political Competition, Cambridge: Harvard University Press.

Saporiti, A. (2008) Existence and uniqueness of Nash equilibrium in electoral competition games: The hybrid case, Journal of Public Economic Theory, 10, 827-857. 